\title{
Palliative Surgery
}

National Cancer Institute

\section{Source}

National Cancer Institute. Palliative Surgery. NCI Thesaurus. Code C17883.

Surgery that does not alter the course of a disease, but improves the quality of life. 